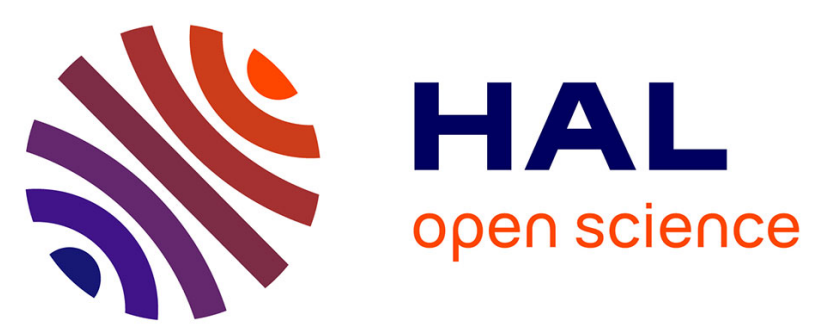

\title{
Uncertainty quantification applied to the radiological characterization of radioactive waste
}

Biagio Zaffora, Matteo Magistris, Gilbert Saporta, Jean-Pierre Chevalier

\section{To cite this version:}

Biagio Zaffora, Matteo Magistris, Gilbert Saporta, Jean-Pierre Chevalier. Uncertainty quantification applied to the radiological characterization of radioactive waste. Applied Radiation and Isotopes, 2017, 127, pp.142-149. 10.1016/j.apradiso.2017.06.001 . hal-01676367

\section{HAL Id: hal-01676367 https://hal.science/hal-01676367}

Submitted on 8 Jan 2018

HAL is a multi-disciplinary open access archive for the deposit and dissemination of scientific research documents, whether they are published or not. The documents may come from teaching and research institutions in France or abroad, or from public or private research centers.
L'archive ouverte pluridisciplinaire HAL, est destinée au dépôt et à la diffusion de documents scientifiques de niveau recherche, publiés ou non, émanant des établissements d'enseignement et de recherche français ou étrangers, des laboratoires publics ou privés. 


\title{
Uncertainty quantification applied to the radiological characterization of radioactive waste
}

\author{
B. Zaffora ${ }^{\mathrm{a}, *}$, M. Magistris ${ }^{\mathrm{a}}$, G. Saporta ${ }^{\mathrm{b}}$, J.-P. Chevalier ${ }^{\mathrm{b}}$ \\ a Radiation Protection Group, European Organization for Nuclear Research, 1211, Geneva 23, Switzerland \\ b CNAM, 292 Rue Saint-Martin, 75003 Paris, France
}

Keywords:

Radioactive waste

Uncertainty quantification

Particle accelerator

Random vector

\begin{abstract}
A B S T R A C T
This paper describes the process adopted at the European Organization for Nuclear Research (CERN) to quantify uncertainties affecting the characterization of very-low-level radioactive waste. Radioactive waste is a by-product of the operation of high-energy particle accelerators. Radioactive waste must be characterized to ensure its safe disposal in final repositories. Characterizing radioactive waste means establishing the list of radionuclides together with their activities. The estimated activity levels are compared to the limits given by the national authority of the waste disposal. The quantification of the uncertainty affecting the concentration of the radionuclides is therefore essential to estimate the acceptability of the waste in the final repository but also to control the sorting, volume reduction and packaging phases of the characterization process. The characterization method consists of estimating the activity of produced radionuclides either by experimental methods or statistical approaches. The uncertainties are estimated using classical statistical methods and uncertainty propagation. A mixed multivariate random vector is built to generate random input parameters for the activity calculations. The random vector is a robust tool to account for the unknown radiological history of legacy waste. This analytical technique is also particularly useful to generate random chemical compositions of materials when the trace element concentrations are not available or cannot be measured. The methodology was validated using a waste population of legacy copper activated at CERN. The methodology introduced here represents a first approach for the uncertainty quantification (UQ) of the characterization process of waste produced at particle accelerators.
\end{abstract}

\section{Introduction}

The present study introduces an approach to estimate uncertainties in radioactive waste characterization.

The characterization of radioactive waste is a complex task, especially when historical waste is involved. At the European Organization for Nuclear Research (CERN), $\gamma$-ray spectrometry is used to estimate the specific activity of easy-to-measure (ETM) radionuclides (IAEA, 2007). Difficult-to-measure (DTM) radionuclides, which are $\beta$ and low-energy $X$-ray emitters, are either measured by radiochemical techniques or evaluated by calculations and Monte Carlo simulations (ISO, 2007, 2013). The specific activities are then compared to the acceptance limits of the national agencies for waste management. Waste producers must ensure that these limits are respected and must estimate the distribution of the quantities of interest together with their uncertainties (Classification, 1994; Lowenthal, 1998).

We present three methodologies to estimate the activity of DTM radionuclides and for each method we describe the associated uncertainty quantification (UQ) strategy. We introduce the application of linear regression, geometric mean and the Mean Activity Method for

\footnotetext{
* Corresponding author.

E-mail address: biagio.zaffora@cern.ch (B. Zaffora).
} 
DTM's activity estimation. Confidence intervals, geometric standard deviation and standard errors are discussed for UQ purposes.

We conclude by presenting an analytical technique to predict the activity of the so-called impossible-to-measure (ITM) radionuclides (ISO, 2013). This technique lies on the construction of a mixed multivariate random variable to stochastically extract activation parameters.

We finally combine the UQ techniques into a global scheme to quantify the uncertainty of the waste characterization process. In the following sections we adopt the terminology presented by the Guide to the expression of uncertainty in measurement (GUM) (JCGM 100, 2008). In this article the term "standard uncertainty" is used to express the standard deviation of a given quantity.

\section{Acceptance and hazard factors}

CERN eliminates its radioactive waste in the repositories of its two Host States, France and Switzerland, in accordance with a tripartite agreement between the Organization and the latter, signed on 15 November 2010 and entered into force on 16 September 2011.

Very-low-level radioactive waste (VLLW) produced at CERN is disposed of in the French repository in the Aube district. The acceptance criteria are based on the hazard factor called IRAS (Radiological Acceptance Index in Storage):

$I R A S=\sum_{i} \frac{a_{i}}{L_{i}}$

where $a_{i}$ is the specific activity of the radionuclide $i$ (in Bq/g) and $L_{i}$ is the specific activity limit of the radionuclide $i$ defined as $L_{i}=10^{\text {Class }_{i}}$. The class of a radionuclide gives information on its radiotoxicity and varies from 0 to 3 (Critères, 2013).

Waste is accepted at the final repository if the IRAS of each package is below 10 and the weighted IRAS of the batch is below 1 :

$I R A S_{\text {batch }}=\frac{\sum_{j} I R A S_{j} \times M_{j}}{\sum_{j} M_{j}}<1$

where $I R A S_{j}$ is the IRAS of the package $j$ as given by Eq. (1) and $M_{j}$ is its weight.

The activity of ETM, DTM and ITM radionuclides are estimated using different techniques. To make explicit the contribution to the IRAS of each one of these families Eq. (1) can be rewritten as follows:

$I R A S=\sum_{l} \frac{a_{E T M, l}}{L_{l}}+\sum_{m} \frac{a_{D T M, m}}{L_{m}}+\sum_{n} \frac{a_{T T M, n}}{L_{n}}$

where the first summation accounts for the specific activity $a_{E T M}$, the second term includes the measured DTM radionuclides and the third summation evaluates the contribution to the IRAS of ITMs.

The next section presents the uncertainty calculation of the terms given in Eq. (3).

\section{Estimation of uncertainties}

\subsection{Uncertainty on ETM radionuclides}

The major contributors to the uncertainty of $a_{E T M}$ are the weight/ density of the waste, the activity distribution within a package, the geometry of the waste items, and the relative position detector/ package. A reasonable sorting, based for example on dose rate ranges, helps to limit the effects of activity hotspots (Rzyski and Suarez, 1988). Compaction of high volume objects and filling with small-sized items are effective techniques to create more uniform waste packages.

We can write the specific activity of ETM radionuclides as a function of various input parameters including the net area of a peak $S_{\text {net }}$ (given by the difference of a gross count and a background count), the weight of the sample or the waste package $m$, the $\gamma$ emission probability $I_{\gamma}$, the counting time $t$, the efficiency calibration $\epsilon$, and the decay correction factor $K$ (Knoll, 2010; Gilmore, 2008):

$a_{E T M}=f\left(S_{n e t}, m, I_{\gamma}, t, \epsilon, K\right)=\frac{S_{n e t}}{m I_{\gamma} t \in K}$.

In this article we limit the discussion to the elements that are of interest for VLLW radiological characterization. A rigorous treatment of uncertainties in $\gamma$-ray spectrometry can be found in Lépy et al. (2015). Complementary information can be found in Sima and Arnold (2009).

If $a_{E T M}$ is estimated from a single peak without interferences (we use the hat notation for estimated quantities; in this case the estimate of the activity of the ETM is represented by $\hat{a}_{E T M}$ ), a simplified formulation of the relative combined standard uncertainty of $\hat{a}_{E T M}$ (or relative standard deviation), indicated with $u_{c}\left(\hat{a}_{E T M}\right) / \hat{a}_{E T M}$, is given by Canberra (2009):

$\frac{u_{c}\left(\hat{a}_{E T M}\right)}{\hat{a}_{E T M}} \approx \sqrt{\left(\frac{u\left(S_{\text {net }}\right)}{S_{\text {net }}}\right)^{2}+\left(\frac{u(m)}{m}\right)^{2}+\left(\frac{u\left(I_{\gamma}\right)}{I_{\gamma}}\right)^{2}+\left(\frac{u(t)}{t}\right)^{2}+\left(\frac{u(\epsilon)}{\epsilon}\right)^{2}+\left(\frac{u(K)}{K}\right)^{2}}$.

Eq. (5) is simplified because assumes independence between the input variables but also because real spectra often present multiple interfering peaks from which the activity is evaluated using numerical methods. A complete formulation of the uncertainty associated to the activity of $\gamma$-emitters, including the evaluation of the covariance terms, can be found in Lépy et al. (2015).

The net counts under the peak, the weight or the counting time in Eq. (5) can be evaluated following classical uncertainty propagation as showed by GUM (JCGM 100, 2008). However, the uncertainty of the efficiency term can be difficult to estimate when characterizing legacy radioactive waste. This is mainly due to the unknown activity and weight distributions within a waste package.

At CERN, in the absence of more precise information, multiple measurements are performed on different sides of the waste package assuming uniform activity/weight distributions. Specific tests would be required to validate the assumption of uniformity and to quantify the extent of the bias affecting the efficiency calibration function. At the same time, operational uniformity checks have been implemented to limit the impact of possible heterogeneities in activity and weight distributions. We can cite for example dose rate screening and sorting of waste, mixing, cutting and compaction of waste items before the packaging and selection of radioactive waste with similar radiological characteristics whenever possible.

When multiple measurements are performed on a waste package, the specific $\gamma$ activity of an ETM radionuclide is computed as the weighted average activity obtained from multiple measurements. The standard deviation of the average ETM activity $u\left(\left\langle a_{E T M}\right\rangle\right)$ obtained from $n$ measurements is given by:

$\frac{1}{u^{2}\left(\left\langle a_{E T M}\right\rangle\right)}=\sum_{i=1}^{n} \frac{1}{u_{i}^{2}\left(\hat{a}_{E T M}\right)}$

where $u_{i}\left(\hat{a}_{E T M}\right)$ is the standard deviation of the ith measured activity as calculated from Eq. (5).

It should be noted that stochastic variations of the efficiency calibration function can be generated by the geometrical settings of the system package/detector. The stochastic variations are due to the uncertainties of the package/detector distance, the apparent density of the waste or the height of the waste within its package. To take these effects into account, we generated multiple efficiency calibration functions by making the input parameters vary within given intervals. Nevertheless, such stochastic variations play a minor role compared with the relative uncertainties affecting the total efficiency (5-10\% according to Lépy et al., 2015).

The extreme case of a highly heterogeneous waste package would require dedicated simulations. However, such case is highly unlikely for legacy metallic waste at CERN where the application of particularly unfavourable activity and weight distributions would lead to an unjustified overestimation of the real activity of a waste package. 
With this respect, we performed a test to estimate the bias term due to the unknown activity and weight distributions. We considered 56 waste packages filled with legacy metallic blocs activated at CERN. We performed $4 \gamma$ measurements on 4 sides of each waste package and we used the weighted average activity of the major $\gamma$-emitter (Co-60) as best estimator of its unknown true activity. We then calculated the percentage relative error of each measurement with respect to the experimental weighted average for every waste package and built a distribution of errors. We found that the median percentage relative error due to activity/weight distributions is $\sim 23 \%$. However, the very test we performed is biased because the true activity and weight distributions are unknown and the efficiency function is evaluated considering the weight and activity uniformly distributed. Complementary information on the disposal of metallic waste at CERN can be found in Zaffora et al. (2017).

\subsection{Uncertainty on DTM radionuclides}

This section describes three methods to quantify the uncertainty of the activity of DTM radionuclides. The UQ methods discussed are specific for linear models, experimental scaling factors and the mean activity method which are commonly employed to estimate the activity of DTM radionuclides in nuclear power plants (IAEA, 2007, 2009; ISO, 2007).

\subsubsection{Linear models}

Linear models can be used to predict the activity of DTMs on waste packages if a correlation exists between the specific activities of DTM nuclides and a major ETM, called the key nuclide (KN), measured on representative samples from the waste population. Linear models can be weighted to account for the uncertainties of the measurements on samples. Only results above the detection limits are suitable to be used.

A basic formula to estimate the activity $\hat{a}_{D T M, i}$ of a DTM radionuclide in the $i$ th waste package is:

$\hat{a}_{D T M, i}=\widehat{\beta}_{0}+\widehat{\beta}_{1} \times a_{K N, i}$

where $\left(\widehat{\beta}_{0}, \widehat{\beta}_{1}\right)$ are the model parameters estimated from the $n$ samples collected from the waste population and $a_{K N, i}$ is the measured activity of the key nuclide in the $i$ th waste package. In this model $\widehat{\beta}_{0}$ is often set to 0 (IAEA, 2009; Zaffora et al., 2016).

The confidence interval of $\hat{a}_{D T M}$ for a given activity $a_{K N}$ of the key nuclide can be used to estimate the standard deviation $u\left(\hat{a}_{D T M, i}\right)$ of the specific activity of the generic DTM in the $i$ th waste package (Olive, 2007):

$u\left(\hat{a}_{D T M, i}\right)=s\left(a_{D T M}\right) \sqrt{\frac{1}{n}+\frac{\left(a_{K N}-\bar{a}_{K N}\right)^{2}}{(n-1) s^{2}\left(a_{K N}\right)}}$

where $n$ is the number of samples, $s\left(a_{\text {DTM }}\right)$ is the estimate of the standard deviation of the residuals (sometimes called residual standard error) of the DTMs given by

$s\left(a_{D T M}\right)=\sqrt{\frac{\sum_{i=1}^{n}\left(a_{D T M, i}-\hat{a}_{D T M, i}\right)^{2}}{n-2}}$

and $s\left(a_{K N}\right)$ is the estimate of the standard deviation of the specific activity of the KN. The term $\bar{a}_{K N}$ represents the average activity of $\mathrm{KN}$. From a formal point of view $\hat{a}_{D T M, i}$ is a linear regression estimator (see chapters 6 and 7 of Cochran, 1963) of the true unknown activity of the DTM radionuclide in the $i$ th waste package and Eq. (8) can be used to quantify its uncertainty.

\subsubsection{Scaling factors and geometric mean}

The experimental scaling factor technique consists of checking for consistent and reproducible correlations between the measured activities of DTM radionuclides and the activity of a key nuclide from a representative sample collected from the waste population. The experimental correlation is used to estimate $a_{D T M}$ by measuring the activity of the KN in each waste package (ISO, 2007; IAEA, 2009).

If activated under similar conditions, the activity distribution of samples is often log-normal. This is commonly observed for both $\gamma$ and $\beta$ emitters. The distribution of the scaling factors, calculated as the ratios of $a_{D T M}$ and $a_{K N}$, is also log-normal (Cochran, 1963).

For log-normally distributed scaling factors a good estimator of central tendency is the geometric mean $\bar{G}_{S F}$ (ISO, 2007):

$\bar{G}_{S F}=\exp \left(\frac{\sum_{i=1}^{n} \ln \left(S F_{i}\right)}{n}\right)=\sqrt[n]{\prod_{i=1}^{n} S F_{i}}$

where $S F_{i}$ for the sample $i$ is the ratio $S F_{i}=a_{D T M, i} / a_{K N}$ (Zaffora et al, 2016).

The uncertainty of the geometric mean $u\left(\bar{G}_{S F}\right)$ can be calculated as the standard error of the geometric mean scaling factor (IAEA, 2009; Norris, 1940; Harding et al., 2014):

$u\left(\bar{G}_{S F}\right)=\bar{G}_{S F} \times \frac{s\left(\ln \left(S F_{i}\right)\right)}{\sqrt{n-1}}$

where $\bar{G}_{S F}$ is given by Eq. (11), $s\left(\ln \left(S F_{i}\right)\right)$ is the estimate of the standard deviation of the natural logarithm of the scaling factors and $n-1$ is the degree of freedom of the sample.

The uncertainty $u\left(\hat{a}_{D T M}\right)$ of the activity of the DTM in the package $i$ $\left(\hat{a}_{D T M, i}=\bar{G}_{S F} \times \hat{a}_{K N, i}\right)$ is calculated propagating $u\left(\hat{a}_{K N, i}\right)$ from Eq. (5) or if the mean over multiple measurements is used, Eq. (6) and the standard error of the geometric mean scaling factor from Eq. (11). As for the linear model, only values above the detection limits should be used to perform this estimation.

The relative combined standard uncertainty of DTM's activity in the $\mathrm{i}^{\text {th }}$ package is:

$\frac{u_{c}\left(\hat{a}_{D T M, i}\right)}{\hat{a}_{D T M, i}}=\sqrt{\frac{u^{2}\left(\bar{G}_{S F}\right)}{\bar{G}_{S F}^{2}}+\frac{u^{2}\left(\hat{a}_{K N, i}\right)}{\hat{a}_{K N, i}^{2}}}$.

In the last equation the mixed term of the general formula of uncertainty propagation is neglected because the correlation between $\bar{G}_{S F}$ and $\hat{a}_{K N}$ is 0 .

\subsubsection{Mean activity method}

The so-called Mean Activity Method is a technique to calculate the specific activity of difficult-to-measure radionuclides if DTMs and the key nuclide are not correlated. This technique consists of calculating the arithmetic average of a sample's activity for each DTM. The calculation includes values below the limit of detection (LOD). The standard uncertainty of the mean can be quantified via the standard error of the mean which is the ratio between the standard deviation of $a_{D T M}$ $\left(s\left(a_{D T M}\right)\right)$ and the square root of the number of collected samples $n$ :

$u_{c}\left(\bar{a}_{D T M}\right)=\frac{s\left(a_{D T M}\right)}{\sqrt{n}}$.

Many algorithms exist when dealing with values below the detection limit (Croghan and Egeghy, 2003). Common replacement methods consist of substituting the limit of detection by $0, L O D / 2, L O D / \sqrt{2}$ or using the limit itself for calculations.

To show an application of this method we consider a sample of 87 items of historical activated copper. The specific activity of Ni-63 was measured via radiochemical analysis. 64 values are above the detection limit. In this example we used the detection limits without substitution. Table 1 shows summary statistics of the activity of Ni-63.

From Table 1 we can see that the mean activity of Ni-63 $(0.53 \mathrm{~Bq} / \mathrm{g})$ is higher than the median activity $(0.50 \mathrm{~Bq} / \mathrm{g})$. This is due to the underlying distribution of Ni-63 activity which is right skewed. Reference IAEA (2009) suggests the use of the mean because this statistics tends to be conservative with respect to the median. The waste producer can however decide which central tendency estimator is more appropriate for the waste population to be disposed of. 
Table 1

Summary statistics of the activity of Ni-63 in a sample of historical copper activated at CERN.

\begin{tabular}{ll}
\hline Statistics & Value $(\mathrm{Bq} / \mathrm{g})$ \\
\hline Minimum & 0.08 \\
1st quartile & 0.27 \\
Median & 0.50 \\
Mean & 0.53 \\
Standard deviation & 0.37 \\
3rd quartile & 0.68 \\
Maximum & 2.31 \\
Skewness & 2.11 \\
Kurtosis & 9.41 \\
\hline
\end{tabular}

When sampling dozens tons of radioactive waste only a limited proportion of the waste population is sampled. A census, like in the previous example, is rarely collected for both technical and financial reasons. Moreover, it is often difficult to ensure a perfect probabilistic sampling because some population's items may not be accessible. Alternative methods, such as the bootstrap (Efron and Tibshirani, 1993), Efron, 1979), can be used in this context to quantify standard uncertainties.

\subsection{Uncertainty on ITM radionuclides}

The specific activity of ITM radionuclides and their uncertainty are estimated using the so-called correlation method (ISO, 2013). This technique consists of calculating the relationship between the measured activity of the Key Nuclide and the specific activity of an ITM radionuclide (Cochran, 1963) using activation studies. The proportionality between a $\mathrm{KN}$ and a ITM is called correlation factor $C F$.

When dealing with legacy waste, limited information is available in terms of the radiological history of the waste, but also in terms of its chemical composition. This is also true for traceable waste for which it is impossible to determine the exact position within an accelerator and the exact irradiation/decay cycle. Moreover, trace and impurity elements are often impossible to quantify within reasonable time and cost.

To encompass these limits, a large number of activation scenarios can be considered. An activation scenario is defined as the unique combination of a chemical composition $\mathbf{C C}$, the energy $E$ of a given accelerator, the position $P$ of a waste item within the accelerator, the irradiation time $T_{i}$ and the decay time $T_{c}$.

When referring to the generic scenario $\mathbf{S}$ we are considering a mixed multivariate random vector in $\mathbb{R}^{n}$ (Rencher, 2002), where the dimension $n$ is the sum of the dimensions of the input parameters:

$\mathbf{S}=f\left(\mathbf{C C}, E, P, T_{i}, T_{c}\right)$.

In the following subsections we discuss how we can generate random chemical compositions and radiological histories for the calculation of correlation factors.

\subsubsection{Chemical composition}

If the quantification of major elements of the chemical composition of a material can easily be performed, the procedure can be extremely long and costly for impurity and trace elements. In fact the quantification of trace elements ends up with a long list of values below the detection limits of the technical apparatus used.

When performing activation studies a number of hypothesis can be made to estimate the amount of trace elements in a chemical composition. This study describes the use of percentiles to build the distribution of trace elements. For major elements normal distributions with narrow confidence intervals can be easily obtained from measurements.

To illustrate the procedure, consider a generic waste made of cathodic copper. This material is often used for general purpose applications such as electric wires.

The number and amount of trace elements in such a material can be highly variable. Common trace and impurity elements are Ag, As, Cd, $\mathrm{Co}, \mathrm{Ni}, \mathrm{Pb}, \mathrm{Sb}$ and $\mathrm{Zn}$ (Delbeke and Rodriguez, 2014). Other trace elements (such as $\mathrm{O}$ and $\mathrm{P}$ ) are not considered because their radiological impacts are negligible in the present context. When experimental data are not available it is possible to collect data from either mining production or industrial sites where estimation of the chemical compositions are often performed. This data can be used to build probability distributions from which we can randomly withdraw a compositional value for each element (ISO, 2013). The distributions so built can be conservative in the sense that the concentrations of trace elements in ores are higher than the concentrations in final copper products. The comparison of the median and mode of the log-normal distributions with the maximum allowed impurities on copper derived products (see the standard ASTM B115-10 of reference ASTM) indicates however that the average statistics of the log-normal distributions are in good agreement with the reference standards.

The technical report of reference Delbeke and Rodriguez (2014) gives the percentiles of the concentration of various chemical elements in cathodic copper. As an example we present the percentiles for the element silver.

Analytical techniques can be used to obtain the best fitting distribution from a set of percentiles. In the present study we are mainly interested in log-normal distributions according to ISO (2013). The probability distribution obtained from the data of Table 2 is shown in Fig. 1. For silver, the reference standard ASTM B115-10 ASTM, indicates that the maximum allowed impurities of silver are $25 \mathrm{ppm}$ for Grade 1 and $70 \mathrm{ppm}$ for Grade 2. Both the mode and the median Ag concentrations showed in Fig. 1 belong within this interval. These values are often below the detection limits of the instruments presently used at CERN. A comparison of the distributions with the data sheets from the producers of the materials employed at CERN can also be used to validate the amount of trace elements in a waste population.

We can proceed in a similar way for each of the other trace elements and obtain a set of distributions from which we can build a random chemical composition. From a mathematical point of view, the $\mathbf{C C}$ is also a mixed multivariate random vector in $\mathbb{R}^{m}$, where $m$ is the number of chemical elements in the material composition.

\subsubsection{Radiological history}

The parameters that determine the radiological history of an item of waste are given by Eq. (14), with the exception of the chemical composition. For legacy waste we can use a similar approach to the one used for the chemical composition to randomly generate a scenario.

In this case we deal with two discrete distributions (Energy and Position within a tunnel) and two continuous distributions (Irradiation and Decay Time). In particular we have:

- 5 beam energies, ranging from $160 \mathrm{MeV}$ for the Linac4 up to $7 \mathrm{TeV}$ for the Large Hadron Collider

- 7 reference positions, from the zone close to the beam impact to the areas behind the concrete shielding

- two continuous uniform distributions of $T_{i}$ and $T_{c}$ that can potentially cover a time frame from 0 to 40 years.

If information about a waste item is known, the extraction of a given scenario can be simplified by exclusions of impossible input conditions.

Table 2

Percentiles of silver in general purpose cathodic copper. The values were calculated from 119 samples collected by the European Copper Institute (Delbeke and Rodriguez, 2014).

\begin{tabular}{lllllll}
\hline Min & $\mathrm{p} 50 \%$ & $\mathrm{p} 60 \%$ & $\mathrm{p} 70 \%$ & $\mathrm{p} 80 \%$ & $\mathrm{p} 90 \%$ & $\mathrm{Max}$ \\
\hline 0.000 & 0.006 & 0.008 & 0.011 & 0.017 & 0.068 & 1.907 \\
\hline
\end{tabular}




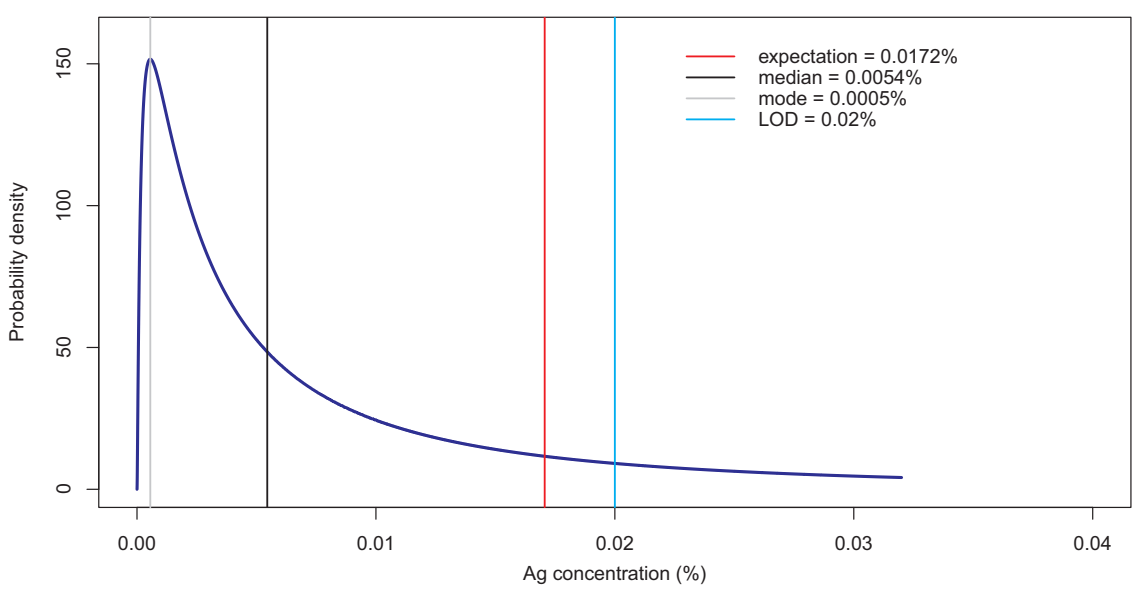

Fig. 1. Log-normal distribution of silver concentration in cathodic copper obtained from percentiles data. We represent the limit of detection (LOD) of common instruments used at CERN together with various central tendency estimators. The population parameters geometric mean $\mu$ and geometric standard deviation $\sigma$ are $(\mu, \sigma)=(-5.21,1.51)$. (For interpretation of the references to color in this figure legend, the reader is referred to the web version of this article.).

\subsubsection{Calculation of the ITM's uncertainty}

When a scenario $\mathbf{S}$ is built we can randomly extract a large number of its realizations (observed values). Each realization is used as an input for either Monte Carlo or analytical calculations. The purpose of simulations and calculations is to establish a complete list of produced radionuclides together with their activities.

For a given scenario $i$ and a given radionuclide $R N_{j}$, the correlation factor $C F_{(i, j)}$ can be calculated as the ratio:

$C F_{(i, j)}=\frac{a_{R N_{(i, j)}}}{a_{K N_{i}}}$

where $a_{R N_{(i, j)}}$ is the calculated activity of the radionuclide $j$ for the scenario $i$ and $a_{K N_{i}}$ is the calculated activity of the key nuclide for the scenario $i$.

We can finally generate a distribution for the $C F$ considering a large number of realizations of the random vector $\mathbf{S}$. According to the distribution obtained for the correlation factor we can calculate the most appropriate central tendency estimator. In practice we can use similar statistical techniques such as the ones used to estimate the scaling factor to evaluate both CFs and the uncertainty $u\left(\hat{a}_{I T M}\right)$ associated to the estimated activity of the ITMs.

We finally recall here that the calculations of the CFs are also useful to predict the range of experimental scaling factors. The comparison of SFs and CFs is also a robust tool to partially validate the consistency of the overall characterization process.

\section{Calculations and experimental results}

We tested the method described in the previous sections on a waste population of historical activated copper. The copper was recovered from electric cables installed within the tunnels of CERN's accelerator complex. The copper was shredded and packaged within 87 drums of average weight $\sim 97 \mathrm{~kg}$. The total batch weight is $\sim 8.5$ tons.

To predict the radionuclide inventory for the waste population we extracted 10,000 realizations from the mixed multivariate random vector $\mathbf{S}$ described in the previous section. The concentration of the chemical element copper was experimentally quantified from a limited number of random samples collected from the waste population and its standard deviation was calculated. A normal distribution is assumed for $\mathrm{Cu}$. Trace elements were randomly extracted from the distributions built according to the method described in Section 3.3.1.

A short list of major radionuclides predicted via simulation, together with their classification as ETM, DTM or ITM, is presented in Table 3.

We further collected 1 composite sample from each sampling unit (drum). The composite sample is a mix of 3 sub-samples taken at 3 different levels of the sampling unit to account for distribution heterogeneities of the shredded copper (Pitard, 1993). Each sample was
Table 3

Short radionuclide inventory including the major contributors to the IRAS when cathodic copper is activated at CERN. The list is built from 10,000 realizations of the random vector $\mathbf{S}$.

\begin{tabular}{lllll}
\hline Radionuclide & $\mathrm{T}_{1 / 2}$ (years) & ETM & DTM & ITM \\
$\mathrm{Co}-60$ & 5.271 & $\checkmark$ & & \\
$\mathrm{Ni}-63$ & 100 & & $\checkmark$ & \\
$\mathrm{H}-3$ & 12.312 & & $\checkmark$ & \\
$\mathrm{Ag}-108 \mathrm{~m}$ & 437.7 & $\checkmark$ & & \\
$\mathrm{Ti}-44$ & 58.9 & $\checkmark$ & & $\checkmark$ \\
$\mathrm{Fe}-55$ & 2.73 & & & $\checkmark$ \\
\hline
\end{tabular}

measured via $\gamma$-spectrometry for the quantification of the activity of the ETM radionuclides. The DTMs Ni-63 and $\mathrm{H}-3$ were quantified via radiochemical analysis. The waste population was also sampled to evaluate the Fe-55 activity although the activity of ITM radionuclides is usually quantified only via calculations (Zaffora et al., 2017).

The measurements performed on the 87 samples show that the Co60 is above the limit of detection in 81 cases. No other major ETM radionuclides were found. For the DTM radionuclides, 64 and 80 samples for, respectively, Ni-63 and H-3 show an activity level above the limit of detection. Only 3 samples of the ITM Fe-55 were quantified above the limit of detection.

We first checked for correlation between the Co-60 and the DTM radionuclides using the Pearson correlation coefficient $r$. Only the pair Co-60/H-3 has a correlation coefficient above $0.5(\mathrm{r}=0.51)$. According to references IAEA (2009) the geometric scaling factor could be used to estimate the activity of $\mathrm{H}-3$. For the pair Co-60/Ni-63 the correlation is below $0.5(r=0.33)$. In this second case the geometric scaling factor is not suitable to be used. Using Eq. (10), we obtained for H-3 an experimental geometric scaling factor $\bar{G}_{S F}^{h 3}=8.67$. The calculation was carried out after the activity of both radionuclides is recalculated at the same reference date $(01 / 07 / 2015)$ and excluding values below the limit of detection. The standard error of the geometric mean scaling factor is calculated according to Eq. (11): $u\left(\bar{G}_{S F}^{h 3}\right)=0.55$; the coverage factor is $\mathrm{k}$ $=1$.

We further built simple linear models to express the relationship between the key nuclide and the DTMs. If the intercept coefficient $\widehat{\beta}_{0}$ of Eq. (7) is set to 0 , we obtain the following relationships: $\hat{a}_{n i 63, i}=11.32 \times a_{c 060, i}\left(R^{2}=0.74\right)$ and $\hat{a}_{h 3, i}=10.53 \times a_{c 060, i}\left(R^{2}=0.50\right)$.

Finally, the Fe-55 could be evaluated using the mean activity method. Fe-55 is however classified as an ITM and we can estimate its activity via calculations (see Section 3.3.3). Using the mean activity method we found $\bar{a}_{f e 55}=0.38 \mathrm{~Bq} / \mathrm{g}$. The standard error of the mean is $0.17 \mathrm{~Bq} / \mathrm{g}$ for a coverage factor $\mathrm{k}=1$. If we use the results from the calculations over the 10,000 realizations of the vector scenario we find a geometric correlation factor $\bar{G}_{C F}^{f e 55}$ equal to 0.054 .

Using the measured activity of the key nuclide Co-60, the 

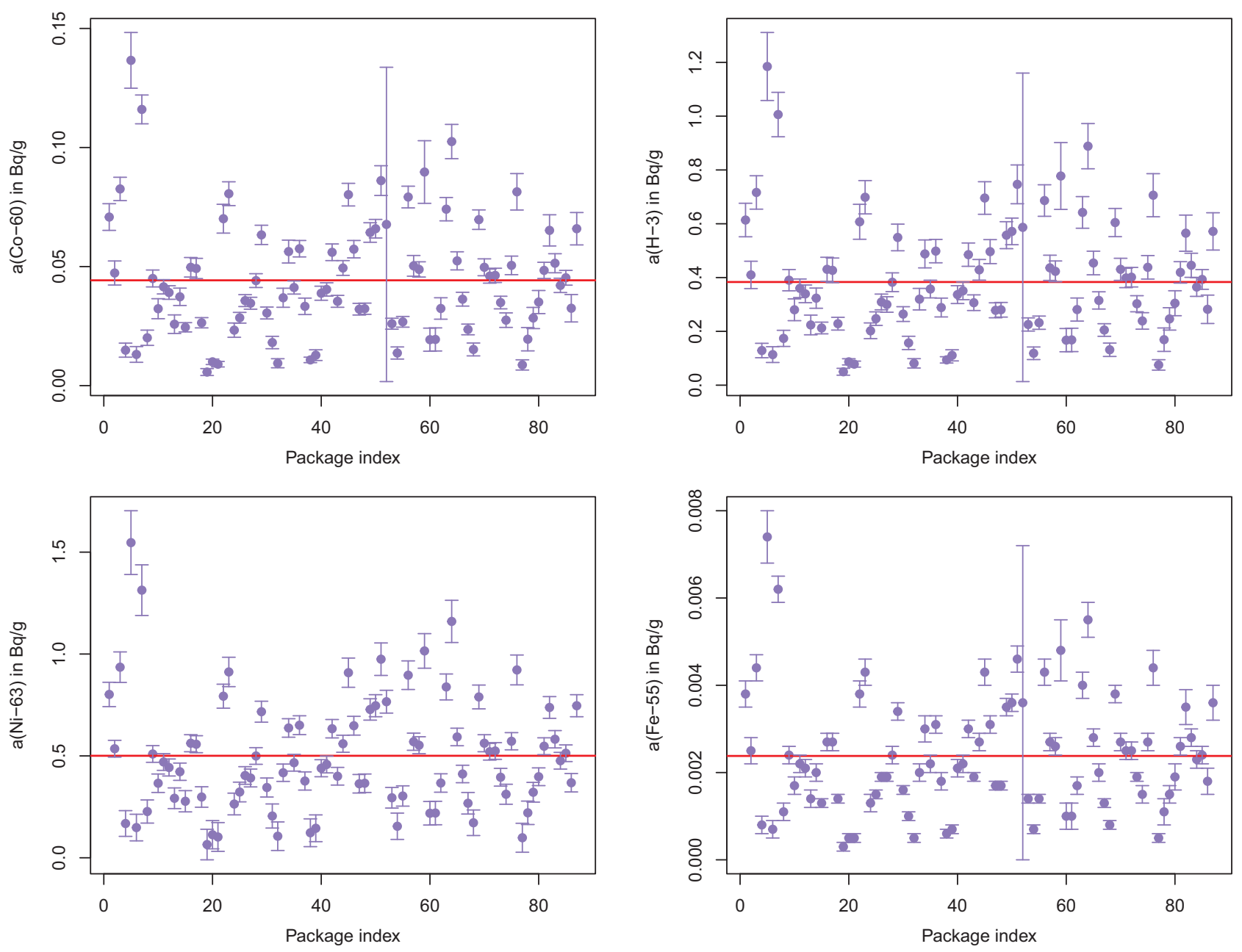

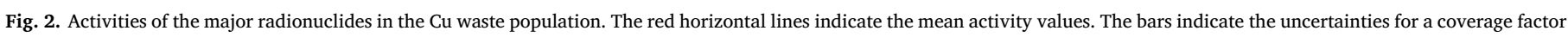
$\mathrm{k}=1$. (For interpretation of the references to color in this figure legend, the reader is referred to the web version of this article.)

experimental geometric scaling factor for $\mathrm{H}-3$, the linear model for $\mathrm{Ni}$ 63 and the correlation factor for Fe-55 we calculated the specific activity of the major radionuclides contained in the waste population. The results are presented in Fig. 2.

From Fig. 2 we can see that the activities of the DTM and ITM radionuclides mimic the same dispersion as the activity of the key nuclide. This is expected because the activities of DTM and ITM radionuclides are obtained from the activity of Co- 60 by multiplication with a constant. We can also see that one container (package of index 52) is characterized by a very high uncertainty of Co-60 activity. This uncertainty affects the uncertainties of $\mathrm{H}-3$ and Fe-55 activities according to Eq. (12). This is not the case for Ni-63 which was evaluated via linear regression (see Eq. (8)).

Table 4 presents selected statistics of the relative standard deviations of the activities of the major radionuclides in the waste population. The coverage factor is $\mathrm{k}=1$.

We finally calculate the IRAS of the waste packages using Eq. (1). The results are presented in Fig. 3. A general formula to express the combined standard uncertainty of the IRAS of a waste package is obtained via uncertainty propagation according to the GUM methodology (JCGM 100, 2008):

$u_{c}^{2}(\operatorname{IRAS})=\sum_{i=1}^{n} \frac{u^{2}\left(a_{i}\right)}{L_{i}^{2}}+\sum_{\substack{i=1 \\ i \neq j}}^{n} \sum_{\substack{j=1 \\ j \neq i}}^{n} \frac{r\left(a_{i}, a_{j}\right) \cdot u\left(a_{i}\right) \cdot u\left(a_{j}\right)}{L_{i} \cdot L_{j}}$

where $r\left(a_{i}, a_{j}\right)$ is the correlation coefficient of the activities of the radionuclides $i$ and $j$.
Table 4

Summary statistics of the relative standard deviation $u_{r e l}$ (in $\%, \mathrm{k}=1$ ) associated to the activities of the major radionuclides in the copper waste population.

\begin{tabular}{lllll}
\hline Statistics & $u_{\text {rel }}\left(a_{c o 60}\right)$ in \% & $u_{\text {rel }}\left(a_{h 3}\right)$ in \% & $u_{\text {rel }}\left(a_{\text {ni63 }}\right)$ in \% & $u_{\text {rel }}\left(a_{f e 55}\right)$ in \% \\
\hline Minimum & 5.2 & 8.2 & 7.1 & 4.8 \\
1st quartile & 7.0 & 9.5 & 7.5 & 7.1 \\
Median & 8.1 & 10.4 & 9.5 & 8.3 \\
Mean & 11.5 & 13.4 & 17.1 & 11.9 \\
SE of the & 1.2 & 1.1 & 2.0 & 1.2 \\
$\quad$ mean & & & & \\
3rd quartile & 12.8 & 14.4 & 16.6 & 12.9 \\
Maximum & 97.5 & 97.7 & 115.4 & 100.0 \\
Skewness & 6.0 & 6.4 & 2.9 & 5.5 \\
Kurtosis & 44.3 & 48.5 & 9.5 & 38.1 \\
\hline
\end{tabular}

The bottom plot of Fig. 3 shows the contribution of the major uncertainty terms to the total budget of uncertainty. The terms contributing less than $0.1 \%$ to the total uncertainty of the IRAS are omitted.

From the top plot of Fig. 3 we can see that the IRAS follows the activity distribution of Co-60 in the waste packages. Similarly, the uncertainty of the IRAS in package 52 is affected by the extremely high uncertainty associated to the activity of the key nuclide. The average relative standard uncertainty of the IRAS is $\sim 11 \%(\mathrm{k}=1)$. The bottom plot of Fig. 3 shows that the uncertainty associated to the activity of the key nuclide has the strongest impact on the standard uncertainty of the IRAS. We found that the average contribution of the uncertainty of Co60 is above $75 \%$. If we add also the contributions of the combined Co- 


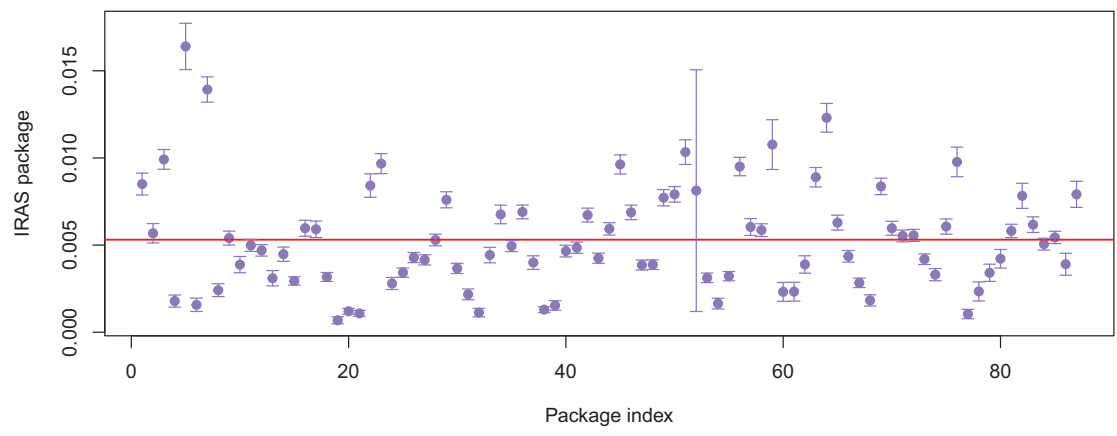

Fig. 3. The top plot shows the IRAS and its standard deviation (k $=1$ ) for the 87 waste packages of very-low-level radioactive copper activated at CERN. The bottom plot illustrates the distributions of the contribution of the uncertainty of each radionuclide's activity to the total IRAS uncertainty. Radionuclides whose activity's uncertainty contributes less than $0.1 \%$ are omitted for clarity.

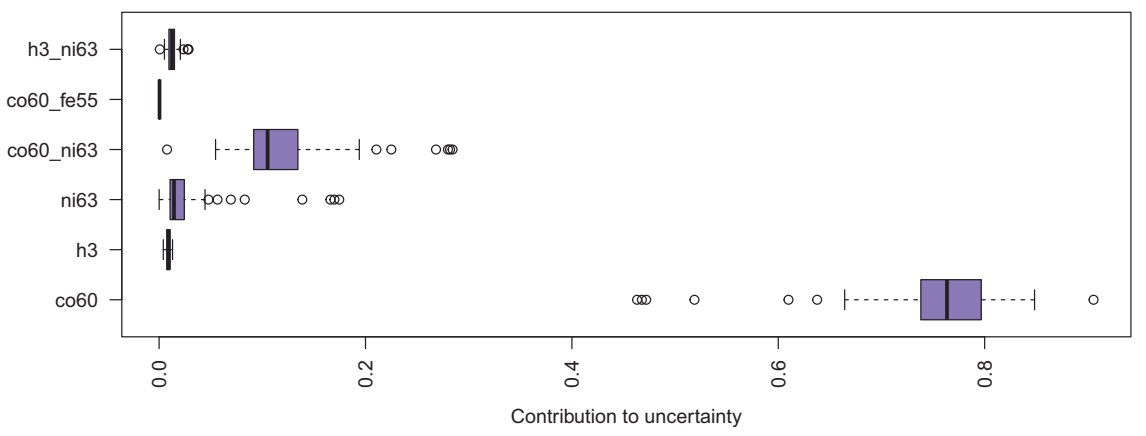

60 terms, the contribution to the total uncertainty of the IRAS adds up to $\sim 90 \%$. For the waste population considered in this study the average contribution of the uncertainties associated to the activities of DTM and ITM radionuclides are below $2.5 \%$.

The reduction of the uncertainty of the IRAS of a waste package of the copper population considered in this study is therefore related to the reduction of the uncertainties associated to the activity of the key nuclide. This consideration however cannot be generalized to other waste populations with a different radiological history. In other cases the activity's uncertainty associated to DTM and ITM radionuclides can play an important role.

We can finally calculate the IRAS batch using Eq. (2) and quantify its uncertainty. A simplified formula to the uncertainty of IRAS $S_{\text {batch }}$ can be obtained assuming that the uncertainties of weight and IRAS of the packages are similar along the waste population. This hypothesis implies that $u\left(M_{i}\right) \simeq u(M)$ and $u\left(I R A S_{i}\right) \simeq u(I R A S)$. This seems a fairly reasonable approximation although some packages may behave differently (see the case of package 52 in our waste population). If this hypothesis holds and we further suppose that the weight and the IRAS of a package are not correlated we can write:

$u_{c}^{2}\left(I_{\left.R A S_{\text {batch }}\right)} \simeq \frac{u^{2}(\operatorname{IRAS})}{n}\left(1+\frac{s^{2}\left(M_{i}\right)}{\bar{M}^{2}}\right)+\frac{u^{2}(M)}{\bar{M}^{2}}\left(\frac{\overline{\operatorname{IRAS}}^{2}}{n}+\frac{s^{2}\left(I R A S_{i}\right)}{n}\right)\right.$

where $n$ is the number of packages in the waste population, $\bar{M}$ and $\overline{I R A S}$ are the average weight and IRAS of the waste packages and $s^{2}\left(M_{i}\right)$ and $s^{2}\left(I R A S_{i}\right)$ are the estimates of the variances of the weight and the IRAS of the waste packages. In Eq. (17) the terms $u^{2}(I R A S)$ and $u^{2}(M)$ are considered constant over the waste population. The calculation of the IRAS for the batch considered gives IRAS $S_{\text {batch }}=2.5 \times 10^{-4}$. The relative standard uncertainty of the $I R A S_{\text {batch }}$ for $\mathrm{k}=1$ is $\sim 1.5 \%$.

\section{Conclusion}

This study describes a procedure to quantify the uncertainty of the main terms of the hazard factor called IRAS, which is needed to evaluate the acceptance of very-low-level radioactive waste by the French repository. The uncertainties for the activity are calculated according to the methodology presented in the Guide to the expression of uncertainty in measurement (GUM).
To quantify the overall uncertainty of the hazard factor, we must estimate the statistical uncertainties and understand if a bias can affect our process. In this article we illustrate how bias can affect the evaluation of ETM radionuclides when the activity or the weight distributions within a waste package are not uniform. A potential bias is also associated with the sampling process to evaluate scaling factors. An unbiased sampling procedure requires that each unit in a waste population has the same probability to be sampled. Such condition can be difficult to implement in special industrial processes where hundred tons of materials are treated.

The waste producer can use conservative values to estimate specific activities. This is the case when not all the units of the waste population are accessible. Such an assumption includes replacing the estimates average specific activity (measured or calculated) with its upper bound or collecting samples at the hotspot. The bias term can increase when such a choice is made and the specific activity can be overestimated. High-biased, conservative approaches are useful to identify preliminary estimators of the hazard factor and can be employed if the uncertainty of the IRAS cannot be calculated using standard GUM procedures.

The characterization procedure presented in this article consists of measuring each waste package via $\gamma$-spectrometry and using the activity of the major $\gamma$-emitter, called key nuclide, to estimate the activity of difficult-to-measure and impossible-to-measure radionuclides. The activity of DTM and ITM radionuclides can be quantified using various techniques such as the geometric mean scaling factor, linear regression, the mean activity method and the correlation factor. A detailed description of the uncertainty quantification process for each technique is described.

We demonstrated that the average contribution of the uncertainty of the key nuclide Co-60 to the overall statistical uncertainty of the IRAS of a waste package is $\sim 75 \%$ for the waste population considered. If we consider the mixed terms of the uncertainty propagation formula, including the key nuclide uncertainty, the amount of explained uncertainty reaches $\sim 90 \%$. The reduction of the uncertainty associated to the IRAS of a waste package of legacy metallic waste produced at CERN is related to the reduction of the uncertainties associated to the activities of major $\gamma$-emitters, such as the key nuclide. However, in some cases the uncertainty of the activity of DTM radionuclides can play a role. This is particularly true when the half-life of DTMs is longer than the half-life of ETMs and the contribution of DTMs increases with the 
decay or storage time of the waste population.

\section{References}

ASTM B115 10. Standard specification for electrolytic copper cathode.

ANDRA. Critères radiologiques d'acceptation des déchets TFA. Technical Report SUR.SP. AMES.02.0007, Chatenay-Malabry, 2013.

Canberra. Customization tools manual. Technical Report Genie 2000 v.3.2, USA, 2009

Cochran, W.G., 1963. Sampling Techniques. John Wiley and Sons, Inc, New York.

Croghan, C.W., Egeghy, P.P., 2003. Methods of dealing with values below the limit of detection using SAS. Presented at the Southeast SAS User Group, pp. 22-24.

Delbeke, K., Rodriguez, H.P., 2014. Copper concentrates. Technical report, European Copper Institute, Avenue de Tervueren 168, 1150 Woluwe-Saint-Pierre, Belgique.

Efron, B., Tibshirani, R.J., 1993. An Introduction to the Bootstrap. Chapman and Hall Inc, London.

Efron, B., 1979. Bootstrap methods: another look at the jackknife. Ann. Stat. 7 (1), 1-26.

Gilmore, G., 2008. Practical gamma-ray spectrometry. John Wiley and Sons, Inc., The Atrium, Southern Gate, Chichester, West Sussex, PO19 8SQ, England.

Harding, B., Tremblay, C., Cousineau, D., 2014. Standard errors: a review and evaluation of standard error estimators using monte carlo simulations. Quant. Methods Psychol. 10 (2), 107-123.

IAEA. Classification of radioactive waste: a safety guide. Technical Report Safety series no. 111-G-1.1, Vienna, 1994.

IAEA. Strategy and methodology for radioactive waste characterization. Technical Report IAEA-TECDOC-1537, Vienna, 2007.

IAEA. Determination and use of scaling factors for waste characterization in nuclear power plants. Technical Report NW-T-1.18, Vienna, 2009.

ISO. ISO 21238. Nuclear energy - Nuclear fuel technology - Scaling factor method to determine the radioactivity of low- and intermediate-level radioactive waste packages generated at nuclear power plants. Geneva, 2007.

ISO. ISO 16966. Nuclear energy - Nuclear fuel technology - Theoretical activation calculation method to evaluate the radioactivity of activated waste generated at nuclear reactors. Geneva, 2013.

JCGM 100:2008. Evaluation of measurement data - guide to the expression of uncertainty in measurement.

Knoll, G.F., 2010. Radiation Detection and Measurement. John Wiley and Sons, Inc, New York.

Lépy, M.C., Pearce, A., Sima, O., 2015. Uncertainties in gamma-ray spectrometry. Metrologia 52 (3), 123-145.

Lowenthal, M.D., 1998. Waste-acceptance criteria and risk-based thinking for radioactivewaste classification. Waste Manag. 18 (4), 249-256.

Norris, N., 1940. The standard errors of the geometric and harmonic means and their application to index numbers. Ann. Math. Stat. 11 (4), 445-448.

Olive, D.J., 2007. Prediction intervals for regression models. Comput. Stat. Data Anal. 51 (6), 3115-3122.

Pitard, F.F., 1993. Pierre Gy's Sampling Theory and Sampling Practice. Heterogeneity, Sampling Correctness, and Statistical Process Control. CRC Press, Boca Raton.

Rencher, A.C., 2002. Methods of Multivariate Analysis. John Wiley and Sons, Inc, New York.

Rzyski, B.M., Suarez, A.A., 1988. Evaluation of homogeneity of radioactive waste forms: statistical criteria. Nucl. Chem. Waste Manag. 8 (3), 211-215.

Sima, O., Arnold, D., 2009. On the Monte Carlo simulation of HPGe gamma-spectrometry systems. Appl. Radiat. Isot. 67 (5), 701-705.

Zaffora, B., Magistris, M., Saporta, G., La Torre, F., 2016. Statistical sampling applied to the radiological characterization of historical waste. EPJ Nucl. Sci. Technol. 2 (34).

Zaffora, B., Magistris, M., Chevalier, J.-P., Luccioni, C., Saporta, G., 2017. A new approach to characterize very-low-level radioactive waste produced at hadron accelerators. Appl. Radiat. Isot. 122, 40-46. 